\title{
1 Bioremediation of industrial pollutants by insects expressing a fungal laccase
}

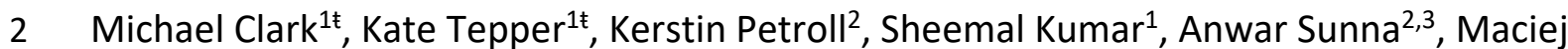

3 Maselko $1,3,4,5 *$

4 Affiliations

$5 \quad{ }^{1}$ Applied BioSciences, Macquarie University, Sydney, NSW, Australia

$6 \quad{ }^{2}$ Department of Molecular Sciences, Macquarie University, Sydney, NSW, Australia

$7 \quad{ }^{3}$ Biomolecular Discovery Research Centre, Macquarie University, Sydney, NSW, Australia

$8 \quad{ }^{4}$ CSIRO Synthetic Biology Future Science Platform, Brisbane, QLD, Australia

$9{ }^{5}$ CSIRO Health and Biosecurity, Canberra, ACT, Australia

$10{ }^{\ddagger}$ Authors contributed equally to this research.

*Correspondence and requests for materials should be sent to maciej.maselko@mq.edu.au

Abstract

Inadequate management of household and industrial wastes pose major challenges to human and environmental health. Advances in synthetic biology may help address these challenges by engineering biological systems to perform new functions such as biomanufacturing of highvalue compounds from low-value waste streams and bioremediation of industrial pollutants. The current emphasis on microbial systems for biomanufacturing, which often require highly pre-processed inputs and sophisticated infrastructure, is not feasible for many waste streams. Concerns about transgene biocontainment have limited the release of engineered microbes or plants for bioremediation. Engineering animals may provide opportunities for utilizing various waste streams that are not suitable for microbial biomanufacturing while effective transgene biocontainment options should enable in situ bioremediation. Here, we engineer the model insect Drosophila melanogaster to express a functional laccase from the fungus Trametes trogii. Laccase expressing flies reduced concentrations of the endocrine disruptor bisphenol $\mathrm{A}$ by more than $50 \%$ when present in their growth media. A lyophilized powder made from engineered adult flies retained substantial enzymatic activity, degrading more than $90 \%$ of bisphenol $\mathrm{A}$ and the textile dye indigo carmine in aqueous solutions. Our results demonstrate that transgenic animals may be used to bioremediate environmental contaminants in vivo and serve as novel production platforms for industrial enzymes. These results support further development of insects, and possibly other animals, as bioproduction platforms and their potential use in bioremediation. 


\section{Main Text}

Developing economically viable approaches for sustainable waste management and mitigation of environmental pollution are important for ensuring a high quality of life ${ }^{1}$. At present, over half of all municipal solid waste is either landfilled ${ }^{2}$, where it is estimated to contribute to $11 \%$ of global methane emissions $s^{3,4}$, or openly dumped, where it contaminates drinking water and disrupts natural ecosystems ${ }^{5}$. Pollution from industrial wastes includes a variety of persistent compounds which have been linked to neurological defects, cancer, and species extinction ${ }^{6-10}$.

Regulatory changes including prohibiting discharge of industrial pollutants and reducing the landfilling of food waste are helpful, however, the cost of compliance can be substantial ${ }^{11}$. Generating value from a variety of waste-streams and developing inexpensive methods to treat pollutants in situ will reduce the costs of achieving sustainability targets and help support a circular economy ${ }^{12}$.

We propose developing solutions by expanding the enzymatic repertoire of select animals to consume waste, degrade xenobiotic chemicals, and produce high-value compounds (Fig. 1). Microbial and fungal enzymes can enable animals to breakdown pollutants in situ. These may include herbivores that degrade pollutants accumulating in plants, fish that secrete enzymes to degrade aquatic pollutants, or invertebrates engineered to interrupt the biomagnification of xenobiotics in food webs. Effective transgene biocontainment safeguards are currently a challenge for plants/microbes ${ }^{13,14}$, but are possible in animals via surgical sterilization or engineered genetic incompatibilities ${ }^{15}$. Developing insects as a biomanufacturing platforms for industrial enzymes, lipids, or other molecules is also an unexplored avenue for waste valorisation.

Insects have advantages over bacteria and fungi for processing many waste-streams. Their complex digestive systems, including mastication, allow them to consume a variety of lowvalue wastes, including municipal organic waste ${ }^{16}$ and livestock manure ${ }^{17}$. Crude waste can be fed directly to insects without pre-processing, refinement, and sterilization necessary for microbial fermentation ${ }^{18,19}$. Some insect species, such as Black soldier flies (Hermetia illucens), which are already used to manage waste have excellent tolerance to bacteria and fungi ${ }^{20}$ and are easily separated from the organic waste for downstream processing ${ }^{21}$. Insect biotechnology is readily scalable for mass production, as the infrastructure can be relatively 
unsophisticated, and the installation costs and land use requirements are low ${ }^{22}$. Furthermore, utilising organic waste means crops that could otherwise be used for food do not need to be diverted as feedstocks for fermentation, and will not threaten food security.

There is a long history of using lepidopteran insect larvae to make recombinant proteins starting with Bombyx mori expressing human $\alpha$-interferon ${ }^{23}$. However, these utilize transient baculoviral systems to infect the larvae. This approach is not practical for large-scale or longterm, multi-generational use. Here we explore the potential of stably engineered insects as platforms for the production of an industrially relevant fungal laccase and bioremediation of polluted sites. Laccases are multicopper oxidases that are present in many taxa. Fungal laccases were selected due to their demonstrated utility for bioremediation of a wide variety of industrial pollutants such as bisphenols ${ }^{24}$, industrial dyes ${ }^{25}$, pharmaceuticals ${ }^{26}$, phenolics ${ }^{27}$, polycyclic aromatic hydrocarbons ${ }^{28}$, perfluoroalkyl and polyfluoroalkyl substances (PFAS) 29,30 , plastics $^{31}$, pesticides ${ }^{32}$, and mycotoxin contaminants such as aflatoxins ${ }^{33}$. Fungal laccases also have diverse industrial applications in the textile, paper and pulp, food, pharmaceutical, chemical synthesis, and forestry industries ${ }^{34-36}$. They have been applied to treat distillery ${ }^{37}$, paper and pulp ${ }^{38}$, and olive mill ${ }^{39}$ industry wastewater. Ligninolytic enzymes, such as fungal laccases, also show potential application in the delignification of feedstocks for biofuel production ${ }^{40}$. However, fungal laccases have not yet reached appreciable commercial scale production. This is because the native hosts for laccases often produce low yields and although this has been improved using heterologous hosts, the production levels are still too low for many commercial applications ${ }^{41}$.

We engineered the common fruit fly (Drosophila melanogaster) to express a laccase from the fungus Trametes trogii. Transgenic flies degraded bisphenol A (BPA), a widespread endocrine disruptor ${ }^{42}$, when present in their diet. We also demonstrate that aqueous solutions of BPA and indigo carmine (IC), a textile dye and aquatic pollutant ${ }^{43}$, can be treated with a lyophilized powder from adult transgenic flies to degrade both compounds. Our results describe the first instance of engineering an animal to perform an ecosystem service by heterologous expression of a fungal enzyme and demonstrate the potential for using transgenic strains of insects for enzyme bioproduction. 


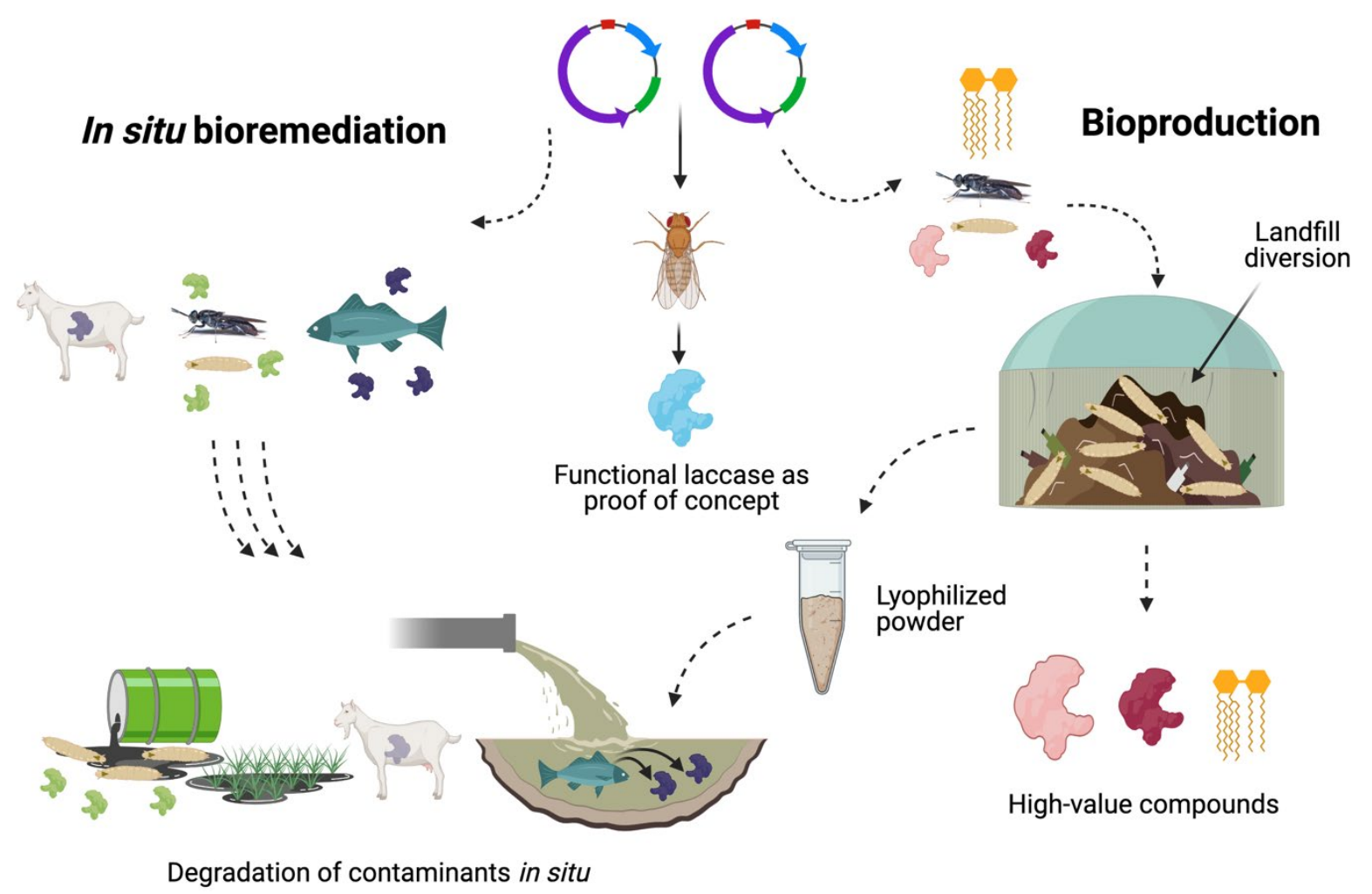

Fig. 1: Applications of transgenic animals for bioremediation and bioproduction.

\section{Results}

$D$. melanogaster and other insects express endogenous laccases involved in immune defense ${ }^{44}$. However, it was unknown if an insect could heterologously express a functional fungal laccase or if its expression would be toxic. We were also uncertain which tissues would provide the optimal expression of a functional laccase for either in vitro or in vivo activity. We selected a short tubulin promoter, previously used to express a toxic gene ${ }^{45}$, with the goal of achieving moderate expression across many tissues. The native fungal signal peptides were replaced with the $D$. melanogaster larval cuticle protein $9^{46}$ signal peptide to facilitate extracellular secretion.

We generated homozygous transgenic strains expressing laccases from two fungal species, $T$. trogii and Polyporus brumalis, known to have a broad substrate range and high redox potential (Table 1). Fly lysates from both were assayed for oxidation of the chromogenic laccase substrate 2,2'-Azino-bis(3-ethylbenzthiazoline-6-sulfonic acid) (ABTS). The 
111

112

113

114

115

engineered $D$. melanogaster strain Dm/Tt.Lcc1, which expresses a laccase from T. trogii, oxidized ABTS (Fig. 2a). There was no activity observed in the wild-type or from the engineered Dm/Pb.Lac1 strain expressing a laccase from P. brumalis (Fig. 2a). Moving forward, we focused on characterizing Dm/Tt.Lcc1.

We next determined if Dm/Tt.Lcc1 flies are capable of degrading bisphenol A (BPA), an endocrine disruptor and emerging environmental contaminant found in many consumer products ${ }^{47}$. BPA has been shown to be a substrate of $T$. trogii laccase when expressed from its native host ${ }^{48}$. We incubated lysates from flies with $25 \mu \mathrm{g} / \mathrm{mL}$ BPA for 21 hours and measured BPA concentrations by liquid-chromatography mass spectrometry (LC-MS). Lysates from Dm/Tt.Lcc1 reduced BPA concentrations by $95 \%$ compared to the no-fly controls and the wild-type fly lysate (Fig. 2b-c).

Table 1. The fungal laccases engineered into $D$. melanogaster

\begin{tabular}{|l|l|l|l|l|}
\hline $\begin{array}{l}\text { Gene } \\
\text { name }\end{array}$ & Native host & $\begin{array}{l}\text { Genbank } \\
\text { accession }\end{array}$ & $\begin{array}{l}\text { Redox potential } \\
(\mathbf{V} \text { vs. NHE, pH 5) }\end{array}$ & Laccase substrates \\
\hline Lcc1 & $\begin{array}{l}\text { Trametes } \\
\text { trogii }\end{array}$ & AJ294820.1 & E॰0.79 V 49 & $\begin{array}{l}\text { Aminophenols, polyphenols, } \\
\text { industrial dyes }\end{array}$ \\
\hline Lac1 52.
\end{tabular}


A. Fly lysate ABTS oxidation

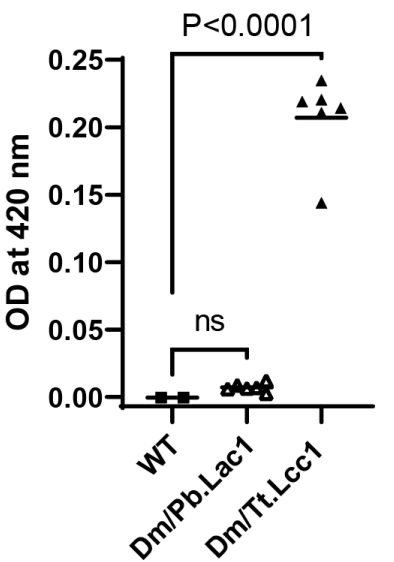

B. Fly lysate BPA oxidation

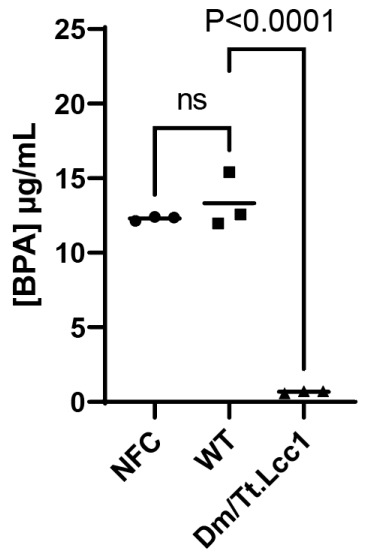

C. Fly lysate representative BPA EIC

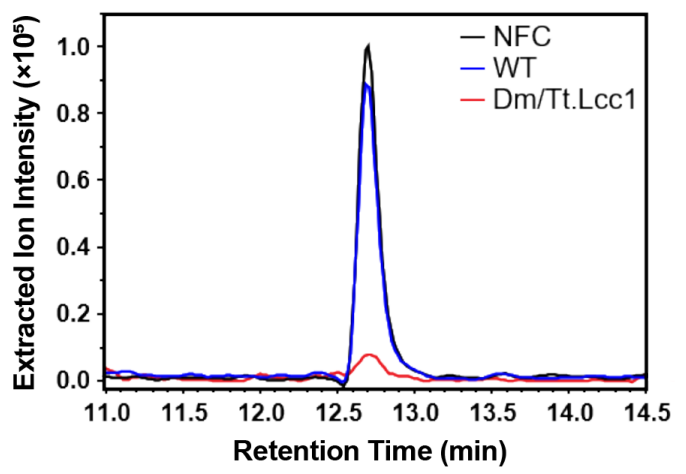

Fig. 2: In vitro laccase substrate oxidation in fly lysates. (A) Fly lysate oxidation of $1 \mathrm{mg} / \mathrm{mL}$ ABTS for 2 hours. $n=2$ biologically independent replicates for WT, $n=6$ for all others. (B) Fly lysate degradation of $25 \mu \mathrm{g} / \mathrm{mL}$ BPA for 21 h. $n=3$ biologically independent replicates. (C) Overlaid representative extracted ion chromatograms (EIC) $(\mathrm{m} / \mathrm{z} 227.15)$ displaying the BPA peak for samples shown in B. Dm/Tt.Lcc1 = D. melanogaster engineered with $T$. trogii laccase, $\mathrm{Dm} / \mathrm{Pb} . \mathrm{Lac1}=D$. melanogaster engineered with $P$. brumalis laccase, WT $=$ wild-type, NFC $=$ No-Fly control. The statistical analysis was conducted using one-way ANOVA using Dunnett's method compared to WT, where ns = not significant.

\section{In vivo laccase activity}

We evaluated the potential of laccase expressing insects to oxidize substrates present in their environment by secreted enzymes. Flies were reared on a minimal media to avoid binding of substrates to insoluble components present in standard media. The minimal media included $1 \mathrm{mM} \mathrm{CuSO}_{4}$ to supply the laccase co-factor. First, we reared 3 adult males and 3 adult females on minimal diet including $1 \mathrm{mg} / \mathrm{mL}$ ABTS for 2 days, after which they were removed. Larvae of the mated flies were grown for an additional 5 days. Dm/Tt.Lcc1 flies oxidized ABTS as indicated by blue colorization of the fly media, however, no oxidation was observed with wildtype flies (Fig. 3a). We controlled for the possibility that the ABTS oxidation was due to laccase that may have been leaking from dead larvae by incubating 6 male flies in ABTS media. After 11 days, only the Dm/Tt.Lcc1 males had oxidized ABTS (Fig. 3b). This result indicates that the oxidation was due to enzyme secreted into the media.

Next, we reared 5 male and 5 female flies in a minimal diet containing $25 \mu \mathrm{g} / \mathrm{mL}$ BPA. Adults were removed after three days. On day 10, BPA was extracted from their diet and quantified by Ultra-High-Performance Liquid Chromatography coupled with fluorescence detection 
152 (UHPLC-FLD). Both Dm/Tt.Lcc1 and wild-type flies had reduced BPA concentrations by 36\% 153 and 45\%, respectively, compared to no-fly controls (Fig. 3c). There was no significant 154 difference between Dm/Tt.Lcc1 and wild-type indicating that the reduction in BPA concentration was not due to the activity of the laccase transgene. The reduction in BPA concentration for wild-type flies relative to the no-fly control may be in part due to BPA

157 absorbed by the adult flies which were removed after three days. Another possibility is that BPA detoxification pathways have been characterized in mammals ${ }^{54}$ and it is possible $D$. melanogaster also contains endogenous pathways capable of detoxifying BPA.

Some laccase substrates, such as ABTS, can function as redox mediators to facilitate the oxidation of additional compounds ${ }^{55}$. When in vivo BPA degradation was tested in the presence of ABTS, Dm/Tt.Lcc1 flies effectively reduced BPA concentration by $68 \%$ and $56 \%$ compared to the no-fly control and wild-type, respectively (Fig. 3d). It is not clear why ABTS was necessary for the in vivo assay and not the in vitro lysate assays. Adding lysate directly to the media also required ABTS for efficient degredation, suggesting that there is some component of the diet which may be interfering (Fig. S1). 


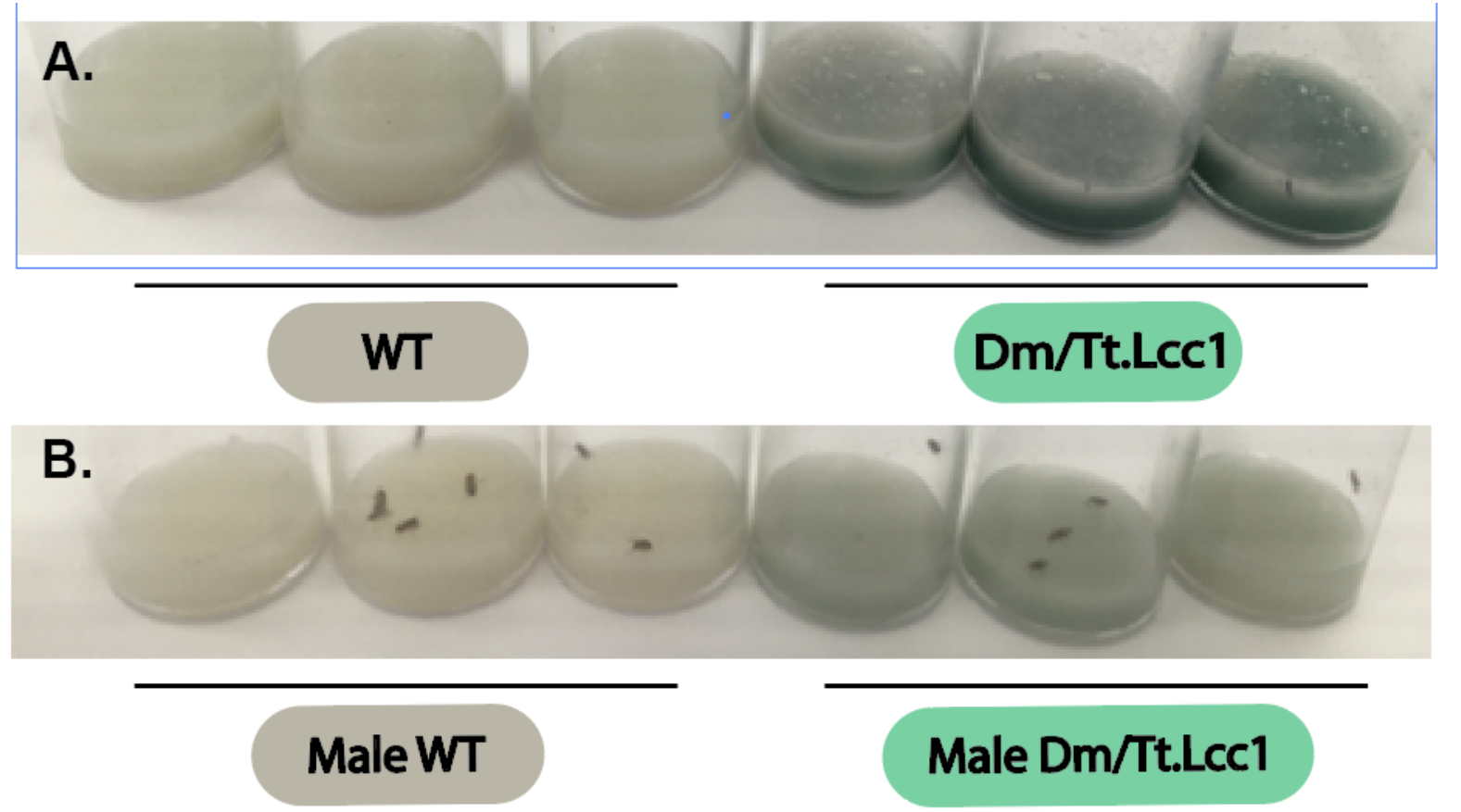

C. In vivo BPA oxidation +BPA -ABTS

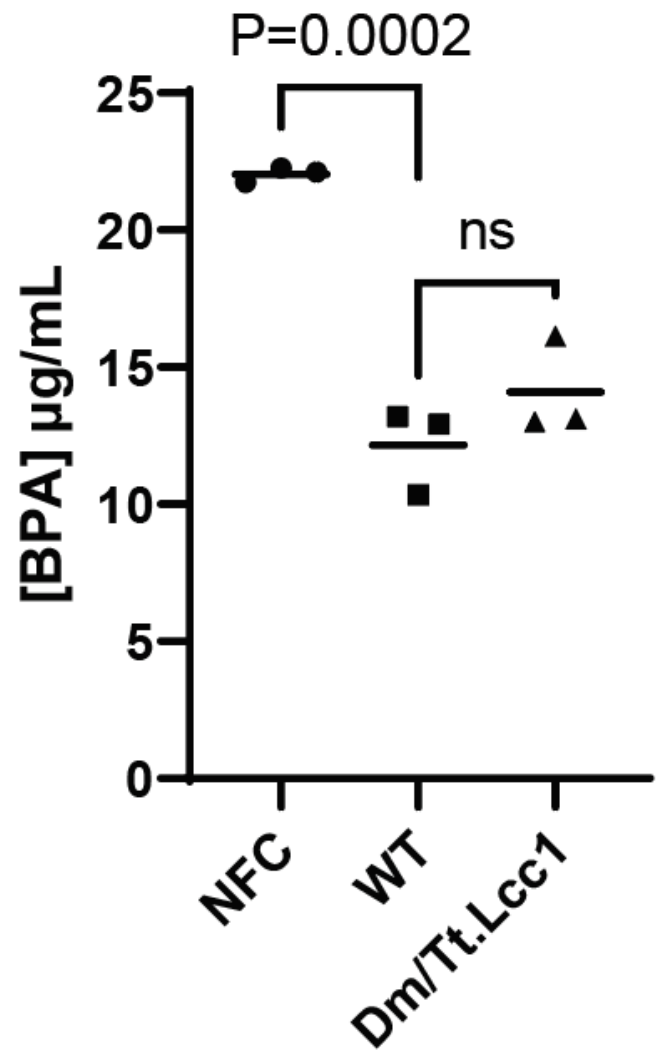
D. In vivo BPA oxidation +BPA +ABTS

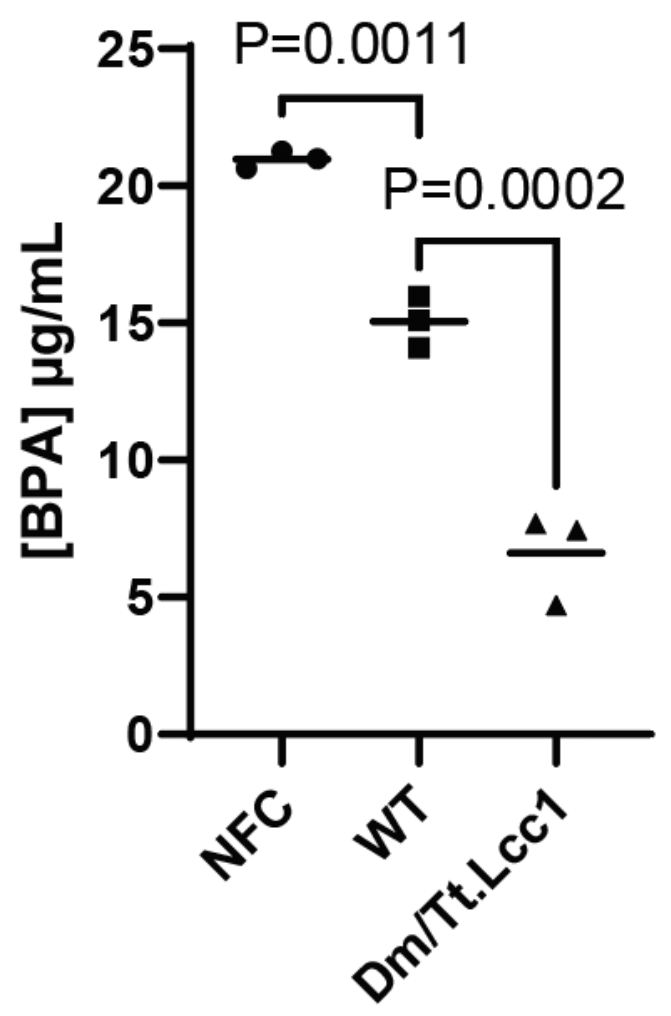

Fig. 3: In vivo laccase enzyme activity. (A) In vivo oxidation of $1 \mathrm{mg} / \mathrm{mL}$ ABTS after 7 days. (B) In vivo oxidation of $1 \mathrm{mg} / \mathrm{mL}$ ABTS after 11 days using only male flies.

(C, D) In vivo degradation of $25 \mu \mathrm{g} / \mathrm{mL}$ BPA without ABTS (C) and with $1 \mathrm{mg} / \mathrm{mL}$ ABTS (D). $n=3$ biologically 
independent replicates. Dm/Tt. Lcc1 $=D$. melanogaster expressing $T$. trogii laccase, $W T=$ wildtype, NFC = No-Fly control. The statistical analysis was conducted using one-way ANOVA using Dunnett's method compared to WT, where ns = not significant.

\section{Lyophilized insect powder laccase activity}

We next examined the potential of insects as platforms to produce a lyophilized laccase which can be easily stored and transported before use. A crude lyophilized powder from Dm/Tt.Lcc1 was compared to a commercially available purified laccase from Trametes versicolor using ABTS as a substrate. The lyophilized Dm/Tt.Lcc1 displayed a total laccase activity of $1.4 \mathrm{U} / \mathrm{g} \pm$ 0.1 SEM dry weight, while the commercial purified laccase displayed a specific activity of 75.3 $\mathrm{U} / \mathrm{g} \pm 6$ SEM dry weight.

We next tested if Dm/Tt.Lcc1 lyophilized whole-fly powder had activity against indigo carmine, a textile industry dye and pollutant ${ }^{43}$. Incubating $5 \mathrm{mg} / \mathrm{mL}$ of Dm/Tt.Lcc1 lyophilized powder in water containing $100 \mathrm{mg} / \mathrm{L}$ indigo carmine resulted in $90 \%$ decolorization after 48 h; however, WT fly lyophilized powder also decolorized $55 \%$ of the dye (Fig. 4a). We observed that insoluble material in the powder appeared to adsorb the dye. However, incubating dye using supernatant from centrifuged lyophilized Dm/Tt.Lcc1 in water did not appear to decolorize indigo carmine compared to controls (Fig. 4b). When the $\mathrm{Cu}^{2+}$ co-factor was included, supernatant from centrifuged lyophilized powder in water decolorized nearly $100 \%$ of indigo carmine in Dm/Tt.Lcc1 and 25\% from WT flies after 90 hours (Fig. 4c-d).

We also evaluated Dm/Tt.Lcc1 lyophilized powder activity against BPA. Incubating $12.5 \mu \mathrm{g} / \mathrm{mL}$ BPA with soluble enzyme from the lyophilized powder in the presence of $\mathrm{Cu}^{2+}$ co-factor resulted in a 96\% reduction of BPA from Dm/Tt.Lcc1 fly powder after 90 hours. Incubation with WT fly powder resulted in a $3 \%$ reduction of BPA (Fig. 4e-f). 
A. IC decolorization (\%) in whole lyophilized powder

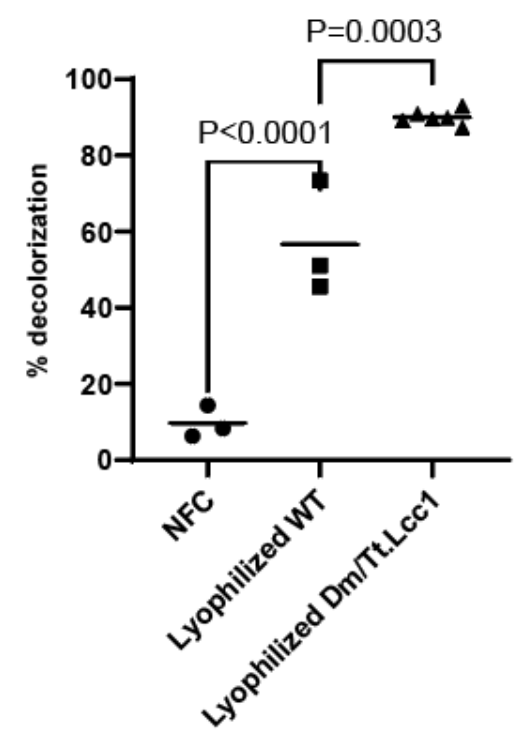

C. Time course IC decolorization (\%) in the soluble fraction of lyophilized powder with copper

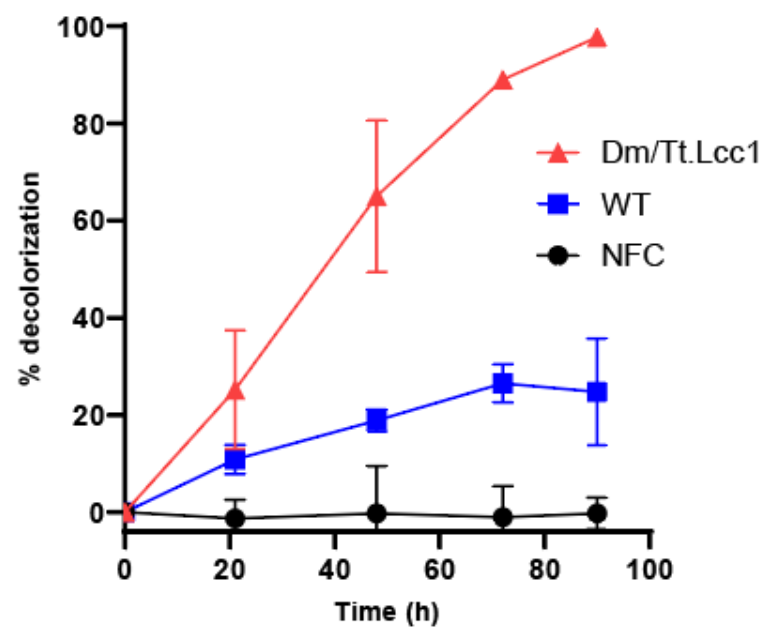

E. Time course BPA oxidation in the soluble fraction of lyophilized powder with copper

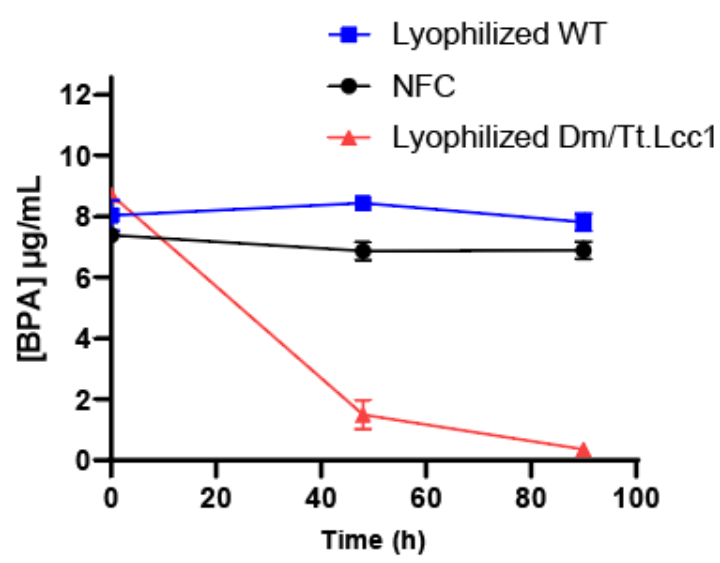

B. Time course IC decolorization (\%) in the soluble fraction of lyophilized powder

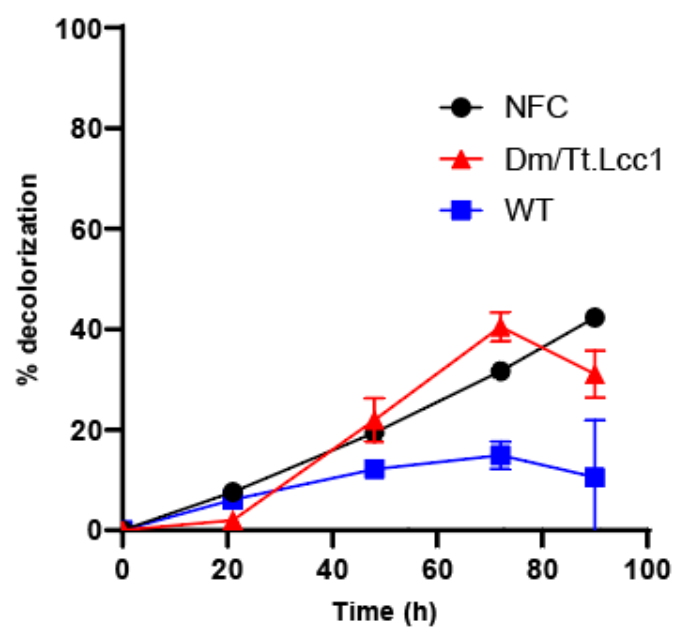

D. IC decolorization (\%) in the soluble fraction of lyophilized powder with copper

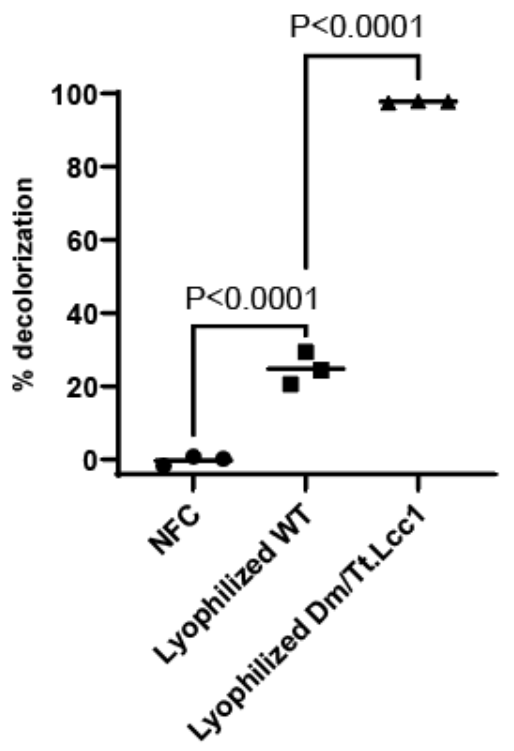

F. BPA oxidation in the soluble fraction of lyophilized powder with copper

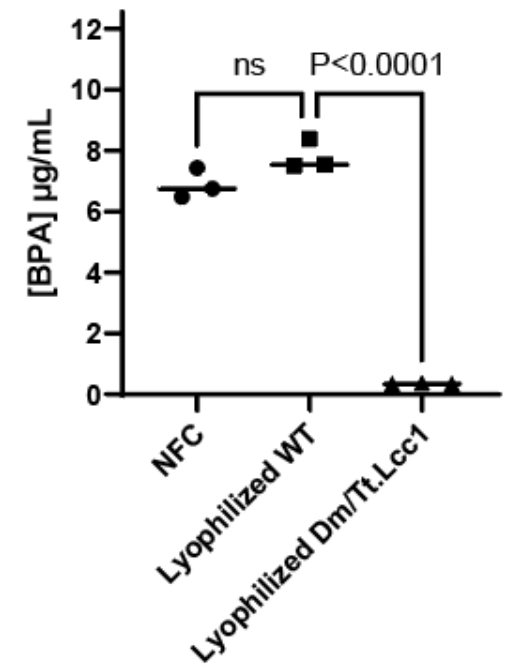


Fig. 4: In vitro activity of lyophilized Dm/Tt.Lcc1 whole-fly powder on laccase substrates. (A)

Decolorization (\%) of $100 \mathrm{mg} / \mathrm{L}$ indigo carmine at $610 \mathrm{~nm}$ in whole lyophilized powder resuspended in water after 48 hours. $n=3$ biologically independent replicates. (B-D) Time course decolorization (\%) of $100 \mathrm{mg} / \mathrm{mL}$ indigo carmine in the soluble fraction of lyophilized powder in water (B) or supplemented with $10 \mathrm{mM} \mathrm{CuSO}_{4}$ (C). Statistical analysis of $\mathbf{C}$ was performed at end point (D). $n=3$ biologically independent replicates. (E,F) Time course degradation of $12.5 \mu \mathrm{g} / \mathrm{mL} \mathrm{BPA}$ in the soluble fraction of lyophilized samples in water supplemented with $10 \mathrm{mM} \mathrm{CuSO}_{4}$ (E). Statistical analysis of $\mathbf{E}$ was performed at the end point (F). $n=3$ biologically independent replicates. Dm/Tt.Lcc1 = D. melanogaster engineered with T. trogii laccase, WT = wild-type, NFC = No-Fly control. The statistical analysis was conducted using one-way ANOVA using Dunnett's method compared to WT, where ns = not significant.

\section{Discussion}

Engineering animals to heterologously express fungal/microbial enzymes may improve sustainable waste management, bioremediation, and facilitate the use of low-value waste streams as inputs for the production of high-value products such as industrial enzymes. Here, we engineer $D$. melanogaster to express a functional laccase from the fungus $T$. trogii. Lysates of this engineered fly (Dm/Tt.Lcc1) oxidized the endocrine disruptor BPA by $95 \%$ in vitro compared to the WT flies. Engineered flies also degraded BPA by $56 \%$ in vivo when added to their diet. Further, a powder prepared from lyophilized engineered flies also degraded BPA by $96 \%$ and the textile dye indigo carmine by almost $100 \%$ in vitro.

Mediator compounds are often used to improve the substrate range and activity of laccases ${ }^{56}$. Although Dm/Tt.Lcc1 lyophilized powder did not require the addition of ABTS as a mediator for degrading BPA, in vivo BPA degradation did. White rot fungi such as Phanerochaete chrysosporium and Pycnoporus cinnabarinus synthesize and secrete endogenous redox mediators ${ }^{57,58}$ and it may be possible to engineer animals to secrete redox mediators to also enhance their bioremediation activities. Alternatively, colloidal lignin or derivatives of lignin also show potential to act as redox mediators ${ }^{59,60}$, and including them may be feasible to assist with in vivo activity.

Using transgenic insects as platforms to convert waste to high-value products, such as industrial enzymes, will require high production titres. Dm/Tt.Lcc1 lyophilized powder made from whole flies had $1.4 \mathrm{U} / \mathrm{g}$ of laccase activity without any attempt at optimization compared 
to $75.3 \mathrm{U} / \mathrm{g}$ of activity from a commercially available purified $T$. versicolor laccase. This is a very promising start and developing tools to genetically manipulate more relevant hosts and strain engineering is needed for insects to reach their potential as bioproduction platforms.

In summary, our findings demonstrate for the first time that insects can be stably engineered to express a functional fungal laccase, which demonstrated in vivo and in vitro laccase activity. Transgenic flies were capable of oxidizing laccase substrates in their environment and they can be used as a lyophilized powder to oxidize substrates in aqueous solutions. These results provide strong support for further research in using transgenic animals as platforms for bioremediation, waste stream management, and recombinant protein production.

\section{Methods}

\section{Construct design and assembly}

NEBuilder ${ }^{\circledR}$ HiFi DNA Assembly Master Mix (New England Biolabs) was used to assemble all constructs. pMBO2744 ${ }^{15}$ was used as a backbone to generate the enzyme expression plasmids. A gBLOCK (IDT $\left.{ }^{\circledR}\right)$ was synthesized that encodes a short alpha-tubulin promoter, a downstream SV40 polyadenylation sequence, and a Notl restriction site in between. This gBLOCK was installed into Notl linearized pMB02744 by HiFi assembly to produce pMC-1-1-1 (Supplementary Table 4). This plasmid was then linearized with Notl, and gBLOCKs that encode for $T$. trogii laccase or $P$. brumalis laccase enzyme were installed with HiFi assembly to generate pMC-1-2-3 and pMC-1-2-6, respectively (Supplementary Table 4). The DNA sequences were codon optimized for expression in $D$. melanogaster using the IDT ${ }^{\circledR} \operatorname{codon}^{-}$ optimization tool. The native fungal signal peptides were replaced with the larval cuticle protein 9 signal peptide. The Kozak sequence, AATCTTACAAA, was upstream of the start codon. See Supplementary Table 2 for plasmid descriptions and Supplementary Table 3 for primers used in this study.

\section{Insect rearing and generation of transgenic fly strains}

Canton S wild-type flies were from the Bloomington Stock Centre (64349, RRID: BDSC 64349). Enzyme expression plasmids were purified using ZymoPURE ${ }^{\mathrm{TM}}$ II Plasmid Midiprep Kit (Zymo Research \#D4200) and sent to BestGene Inc (Chino Hills, Ca) for $\phi C 31$ mediated integration. All strains were maintained on a cornmeal diet based on the Bloomington standard Nutri-Fly formulation (catalog number 66-113; Genesee Scientific). In preparation for the in vitro or in 
vivo laccase enzyme activity assay, the diet for both the transgenic and wild-type strains was supplemented with $\mathrm{CuSO}_{4} \bullet 5 \mathrm{H}_{2} \mathrm{O}(1 \mathrm{mM}$, Merck, CAS-no: 7758-99-9). Flies were reared in a controlled environment room at $25.0^{\circ} \mathrm{C}, 75 \%$ humidity, and a 12 hour light/dark cycle with a 30 minute transition period. See Supplementary Table 1 for all of the fly strains used in this study. Biosafety approval was granted by the Institutional Biosafety Committee of Macquarie University (\#5049).

\section{In vitro enzyme activity assay}

To obtain a fly lysate, ten frozen adult flies were homogenized with a motorized pestle in the stated buffer supplemented with $10 \mathrm{mM} \mathrm{CuSO} \cdot \bullet 5 \mathrm{H} 2 \mathrm{O}$ and acid-washed glass beads. The samples were then agitated along a tube rack 10 times. Each tube was incubated for 15 minutes at $25^{\circ} \mathrm{C}$ and centrifuged twice to remove insoluble debris.

For the ABTS oxidation assay, fly lysates were prepared in citrate-phosphate buffer $(\mathrm{pH} \mathrm{5})$ and added to $1 \mathrm{mM} \mathrm{ABTS}$. The tubes were incubated at $25{ }^{\circ} \mathrm{C}$ for 2.5 hours. The reaction was quenched by adding 5\% trichloroacetic acid (TCA, Sigma Aldrich, catalogue \#T0699, CASno:76-03-9). A UV-Vis spectrophotometer (Jasco V-760 UV-Vis spectrophotometer) was used to measure the absorbance at $420 \mathrm{~nm}$. The measurements were blanked to an equivalent sample that did not contain ABTS.

For the BPA degradation assay, fly lysates or controls were prepared in sodium acetate buffer ( $\mathrm{pH} \mathrm{5}$ ) and added to $25 \mu \mathrm{g} / \mathrm{mL}$ BPA. The assay mixtures were incubated at room temperature for 21 hours. The reaction was quenched by adding 5\% (v/v) trichloroacetic acid (Sigma Aldrich, catalog \#T0699, CAS-no:76-03-9). BPA concentration was analyzed by LC-MS as described below.

\section{In vivo laccase activity assays}

Minimal media:

The minimal media components were for $100 \mathrm{~mL}: 1.58 \mathrm{~g}$ yeast, $0.66 \mathrm{~g}$ agar, $5 \mathrm{~g}$ sugar, $4 \mathrm{~mL} 1$ M propionic acid (pH 4.4), $12.4 \mathrm{mg} \mathrm{CuSO}{ }_{4} \bullet 5 \mathrm{H}_{2} \mathrm{O}$ (1 mM final concentration). A buffer of 20 $\mathrm{mM}$ citrate-phosphate ( $\mathrm{pH} \mathrm{5)}$ was added for the initial ABTS assays, $25 \mathrm{mM}$ sodium acetate buffer (pH 5) was used for all BPA experiments.

ABTS only in vivo experiments: 
To minimal media $100 \mathrm{mg}$ ABTS ( $1 \mathrm{mg} / \mathrm{mL}$ final concentration) was added. Three female and three male adult flies were incubated on the media for 48 hours, after which they were removed. For the all-male ABTS assay, the adult flies remained in the vial for 11 days. The appearance of the dark green radical cation was monitored and recorded with photographs.

For the ABTS + BPA experiments:

To minimal media, BPA was added $(25 \mu \mathrm{g} / \mathrm{mL}$ final concentration). ABTS ( $1 \mathrm{mg} / \mathrm{mL}$ final concentration) was added for the +BPA, + ABTS condition.

For each condition, the liquid media was pipetted into a $14 \mathrm{~mL}$ polypropylene round-bottom culture tube. 5 male and 5 female adult flies were incubated on the media for 3 days and then removed. For the lysate condition, $50 \mu \mathrm{L}$ of lysate from 100 flies was added to the tube. After a total of 10 days the enzymatic reaction was stopped with TCA ( $5 \% \mathrm{v} / \mathrm{v}$ final concentration). The media was melted in a water bath, after which methanol (making $~ 1: 1$ methanol: aqueous TCA for a two-fold dilution of BPA) was added, followed by vortexing. The tubes were then incubated at $300 \mathrm{rpm}$ and $37^{\circ} \mathrm{C}$ for 30 minutes. After incubation, the tubes were centrifuged twice with the final supernatant reserved for BPA quantification by UHPLC-FLD as described below.

\section{Lyophilized Dm/Tt.Lcc1 enzyme activity assays}

Frozen Dm/Tt.Lcc1 or wild-type flies were lyophilized (Alpha 1-4 LDplus, Christ) at $-45^{\circ} \mathrm{C}$ at $0.1 \mathrm{mBar}$. The freeze-dried flies were homogenized in a porcelain mortar and pestle and stored at $4{ }^{\circ} \mathrm{C}$.

For the activity assays, lyophilized Dm/Tt.Lcc1 and commercial T. versicolor laccase (SigmaAldrich, Catalogue 38429) were resuspended in $25 \mathrm{mM}$ sodium acetate buffer (pH 5) supplemented with $10 \mathrm{mM} \mathrm{CuSO}_{4} \bullet 5 \mathrm{H}_{2} \mathrm{O}$. Lyophilized Dm/Tt.Lcc1 did not dissolve, and the soluble fraction was extracted at $25{ }^{\circ} \mathrm{C}$ for 15 minutes at $800 \mathrm{rpm}$ and centrifuged twice to remove the insoluble debris. Enzyme serial dilutions were added in duplicate to $1 \mathrm{mM} A B T S$ and ABTS oxidation was monitored on a PHERAStar FS plate reader (BMG Labtech) at $420 \mathrm{~nm}$ at $25^{\circ} \mathrm{C}$. One unit of enzyme activity was defined as the amount of enzyme that catalyzes the oxidation of $1 \mu \mathrm{mol}$ ABTS $\mathrm{min}^{-1}$ using the molar extinction coefficient $36 \mathrm{mM}^{-1} \mathrm{~cm}^{-1} 61$. 
For the indigo carmine decolorization assay, $5 \mathrm{mg} / \mathrm{mL}$ of lyophilized powder or controls in Millipore water were added to $100 \mathrm{mg} / \mathrm{L}$ indigo carmine (Sigma-Aldrich catalog 131164) and incubated at RT. At 0 and 48 hours, indigo carmine decolorization was monitored on a PHERAStar FS plate reader (BMG Labtech) at $610 \mathrm{~nm}$.

As indigo carmine appeared to adsorb to the lyophilized powder, the soluble fraction was tested for enzymatic activity. $5 \mathrm{mg} / \mathrm{mL}$ lyophilized powder or controls in Millipore water supplemented with and without $10 \mathrm{mM} \mathrm{CuSO}_{4} \bullet 5 \mathrm{H}_{2} \mathrm{O}$ were extracted at $25{ }^{\circ} \mathrm{C}$ for 15 minutes at $800 \mathrm{rpm}$. The samples were centrifuged twice to remove the insoluble debris. The supernatant of the lyophilized powder and controls were added to $100 \mathrm{mg} / \mathrm{L}$ indigo carmine and incubated at room temperature. Indigo carmine decolorization was monitored on a PHERAStar FS plate reader (BMG Labtech) at $610 \mathrm{~nm}$ at 0, 21, 48, 72, and 90 hours.

For the BPA degradation assay, the soluble fraction of the lyophilized powder was extracted as described in the indigo carmine assay. The supernatant of the lyophilized powder and controls were added to $12.5 \mu \mathrm{g} / \mathrm{mL} \mathrm{BPA}$ and incubated at room temperature. At 0,48 , and 96 hours, $100 \mu \mathrm{L}$ aliquots were removed and the reaction was stopped using a $3 \mathrm{KDa}$ nominal molecular weight limit (NMWL) centrifuge filter (Merck Amicon Ultra $0.5 \mathrm{~mL}$ Centrifugal Filters, UFC500396). BPA concentration was analyzed by UHPLC-FLD as described below.

\section{BPA quantification}

BPA in samples was either quantified by UHPLC-FLD analysis or by LC-MS. UHPLC-FLD analysis was performed using an Agilent RRHD ZORBAX Eclipse Plus C18 column (3.0 x $150 \mathrm{~mm} 1.8 \mu \mathrm{m}$, p/n 959759-302) equipped with a UHPLC Eclipse Plus C18 Guard Column, (2.1 mm $1.8 \mu \mathrm{m}$, $\mathrm{p} / \mathrm{n}$ 821725-901). The isocratic mobile phase consisted of acetonitrile (ACN):10 mM KH $\mathrm{PO}_{4}$ $(50: 50 \mathrm{v} / \mathrm{v})$ at a flow rate of $0.5 \mathrm{~mL} / \mathrm{min}$. A gradient wash step was introduced after each run with eluent $\mathrm{B} 100 \% \mathrm{ACN}$ and eluent $\mathrm{A} 10 \mathrm{mM} \mathrm{KH}_{2} \mathrm{PO}_{4}$, starting with $45 \% \mathrm{~B} 1$ from 0 - 1 minute, $70 \%$ B from $1-6$ minutes, 5\% B from $6-11$ minutes, and finally 50\% B from 11 - 16 minutes at $0.3 \mathrm{~mL} / \mathrm{min}$. BPA was detected by setting FLD excitation wavelength at $229 \mathrm{~nm}$, and emission wavelength at $316 \mathrm{~nm}$ and a gain setting of 12. Linear regression of 7-point standards from $12.5 \mathrm{ng} / \mathrm{mL}$ to $12,500 \mathrm{ng} / \mathrm{mL}$ BPA was performed to calibrate BPA concentration. All samples and standards were either filtered using syringe filters (Filtropur $\mathrm{S}$ $0.2 \mu \mathrm{m}$, Sarstedt, 83.1826.001) or centrifuge filters (Merck Amicon Ultra 0.5mL Centrifugal Filters, UFC500396) prior to injection into the UHPLC. 
LC-MS validation and quantification of BPA was performed externally at the Mass Spectrometry Facility at the University of Sydney. LC separation was performed using a Waters Sunfire C18 column ( $5 \mu \mathrm{m}, 2.1 \mathrm{~mm}$ ID x $15 \mathrm{~mm}$ ) coupled to a Bruker amaZon SL mass spectrometer. Gradient elution was performed with a mobile phase consisting of $(A)$ methanol and (B) water with $0.1 \%$ acetic acid at a flow rate of $0.3 \mathrm{~mL} / \mathrm{min}$. The gradient consisted of $20 \%$ A from 0 - 9.3 minutes, $76 \%$ A from 9.3 - 17.3 minutes, $92 \%$ A from $17.3-$ 26 minutes, and finally $20 \%$ A from 26 - 35 minutes. MS analysis was performed using Atmospheric Pressure Chemical Ionisation (ACPI) under positive and negative ionization mode over a range of $200-1400 \mathrm{~m} / \mathrm{z}$. The operation parameters were: Nebulizer 27.3 psi, Dry Gas $4.0 \mathrm{~L} / \mathrm{min}$, Dry temperature $180{ }^{\circ} \mathrm{C}, \mathrm{APCl}$ Vaporisation temperature: $400{ }^{\circ} \mathrm{C}, \mathrm{APCl}$ Corona needle current: $6000 \mathrm{nA}$, Mass mode: enhanced resolution, SPS (Smart parameter setting) Target mass $150 \mathrm{~m} / \mathrm{z}$, compound stability $100 \%$. Linear regression of 6-point BPA standards from $20 \mathrm{ng} / \mathrm{mL}$ to $10 \mu \mathrm{g} / \mathrm{mL}$ BPA was performed to calibrate BPA concentration. An internal deuterated $B P A-D_{16}$ standard was spiked into the samples prior to injection to calibrate ionization in the sample matrix compared to the standard curve matrix prepared in methanol. All samples were centrifuge filtered (Merck Amicon Ultra $0.5 \mathrm{~mL}$ Centrifugal Filters, UFC500396) prior to injection into the LC-MS.

\section{Statistical analysis}

Figures were generated in Prism 9 by GraphPad. For statistical analysis, a one-way ANOVA using Dunnet's method was conducted. The significance level for the analysis was 0.05 , and all error bars represent the standard error of the mean. The exact $P$ values, statistical tests used, and sample numbers $(n)$ can be found in the figure legend.

\section{Acknowledgements:}

We are grateful Dr. Nick Proschogo from the Mass Spectrometry Facility at the University of Sydney. Funding: This material is based upon work supported by the International Technology Center Pacific (ITC-PAC) under Contract No. FA520920P0100, a Macquarie University seeding grant, and the CSIRO Synthetic Biology Future Science Platform. Author Contributions: MC, KT, and MM conceived of this study. MC, KT, KP, AS, and MM designed experiments and analyzed data. MC, KT, KP, and SK performed the experiments. All authors contributed to writing the manuscript. Competing Interests: The authors have filed a patent 

upon request.

382

\section{Supplementary Materials:}

Supplementary Figure 1

Supplementary Tables 1-4

Supplementary Data File

\section{Supplementary information}

\section{In vivo BPA oxidation conditions with lysate}

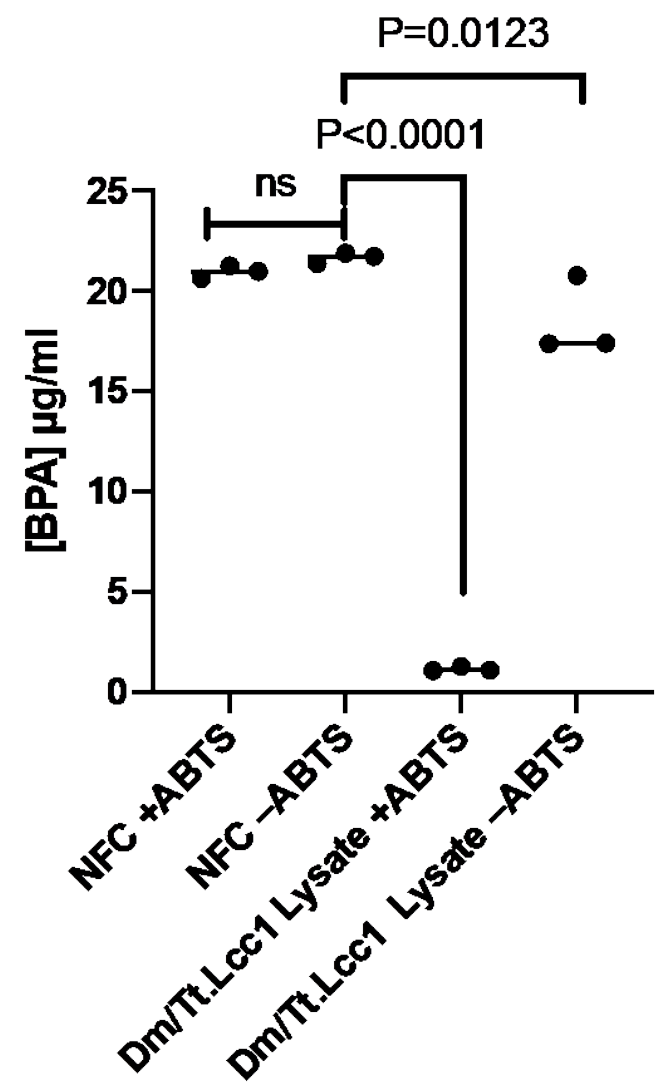

Supplementary figure 1: In vivo BPA degradation conditions comparing Dm/Tt.Lcc1 lysate to NFC. Fly lysate was added to media that contained $25 \mu \mathrm{g} / \mathrm{mL}$ BPA with and without $1 \mathrm{mg} / \mathrm{mL}$ ABTS. Dm/Tt.Lcc1 lysate was unable to degrade BPA without ABTS. $n=3$ biologically independent replicates. The statistical analysis was a one-way ANOVA with Dunnett's method. ns= not significant, Dm/Tt.Lcc1 $=D$. melanogaster engineered with $T$. trogii laccase, $\mathrm{NFC}=$ no fly control. 
Supplementary Table 1: D. melanogaster strains used in this study

\begin{tabular}{|c|c|c|c|}
\hline Strain & Description & Reference & BDSC \# \\
\hline Wild Type & Canton S & 62 & 64349 \\
\hline $2^{\text {nd }}$ Chr. attP3 & $\mathrm{y}^{1} \mathrm{w}^{1118 ;}$ PBac $\{\mathrm{y}[+]-\operatorname{attP}-3 \mathrm{~B}\}$ VK00037 & 63 & 9752 \\
\hline $\begin{array}{l}2^{\text {nd }} \text { Chr. Bal, } \\
\text { Tm2/Tm6b }\end{array}$ & $\mathrm{w}^{1118} ; \mathrm{Gla} / \mathrm{CyO} ; \mathrm{Tm} 2 / \mathrm{Tm} 6 \mathrm{~b}$ & This study & \\
\hline $\begin{array}{l}2^{\text {nd }} \text { Chr. Bal, } \\
\operatorname{Tm} 2 / \operatorname{Tm} 6 b\end{array}$ & w1118; Bl/CyO; Tm2/Tm6b & This study & \\
\hline Dm/Tt.Lcc1 & $\mathrm{y}^{1} \mathrm{w}^{1118 ;}$ PBac\{Tt.Lcc1\}attP-3B & This study & \\
\hline Dm/Pb.Lac1 & $\mathrm{y}^{1} \mathrm{w}^{1118 ;}$ PBac $\{$ Pb.Lac1\}attP-3B & This study & \\
\hline
\end{tabular}

\section{Supplementary Table 2: Plasmids used in this study}

Integrated in Fly
Plasmid

pMB02744 NA
pMC-1-1-1

pMC-1-2-3 Dm/Tt.Lcc1

pMC-1-2-6

Dm/Pb.Lac1
Contains a mini-white gene for $D$. melanogaster selection, an AttB site for genomic integration, and an ampicillin resistance cassette for cloning in E. coli.

Empty vector, with short alphatubulin promoter and SV40 regulatory sequences Short alpha-tubulin promoter driving expression of $L c c 1$ from Trametes trogii

Short alpha-tubulin promoter driving expression of Lac1 from Polyporus brumalis
Reference/

GenBank

Accession \#

Supplementary table 3: Primers used in this study

\begin{tabular}{|l|l|l|}
\hline Name & Sequence $\left.\mathbf{( 5}^{\prime} \rightarrow \mathbf{3}^{\prime}\right)$ & Notes \\
\hline 1stconstruct_F_2.0 & GCTTGGATTTCACTGGAACTAG & pMC-1-1-1 sequencing \\
\hline 1stconstruct_R & TCGAGACCGTGACCTACATC & pMC-1-1-1 sequencing \\
\hline 2ndconstruct_F_2.0 & cgtttgtcaagcctcatagc & $\begin{array}{l}\text { pMC-1-2-3/pMC-1-2-6 } \\
\text { sequencing }\end{array}$ \\
\hline 2ndconstruct_R_2.0 & ttcattttatgtttcaggttcaggg & $\begin{array}{l}\text { pMC-1-2-3/pMC-1-2-6 } \\
\text { sequencing }\end{array}$ \\
\hline P.bru_F & TGACTTTCAGGTGCCCGATC & pMC-1-2-6 sequencing \\
\hline P.bru_R & GCCGGGAATGTGAGTTCGATG & pMC-1-2-6 sequencing \\
\hline
\end{tabular}




\begin{tabular}{|l|l|l|}
\hline T.tro_F & ATGGCCCTGCATTCGTCAAC & pMC-1-2-3 sequencing \\
\hline T.tro_R & CGTTGCTGGCAAGCTGATTTC & pMC-1-2-3 sequencing \\
\hline
\end{tabular}

\begin{tabular}{|c|c|}
\hline \multicolumn{2}{|c|}{ Supplementary table 4: gblocks used to construct laccase expression plasmids: } \\
\hline $\begin{array}{l}\text { Name and } \\
\text { description }\end{array}$ & Sequence $\left(5^{\prime} \rightarrow 3^{\prime}\right)$ \\
\hline $\begin{array}{l}\text { Gblock that } \\
\text { includes short } \\
\text { alpha-tubulin } \\
\text { and SV40 } \\
\text { regulatory } \\
\text { sequences for } \\
\text { expression of } \\
\text { the enzymes in } \\
\text { Drosophila } \\
\text { melanogaster. } \\
\text { Contains a Notl } \\
\text { site between } \\
\text { the promoter } \\
\text { and terminator } \\
\text { for assembly of } \\
\text { enzyme gene } \\
\text { gblocks. } \\
\text { Generates pMC- } \\
1-1-1 \text { from } \\
\text { pMBO2744 }\end{array}$ & $\begin{array}{l}\text { AGCGTAAGCTTCGTACGTAGCGACCGTCTCAAAGTACTGCCTTTCTGCGTTGGAAAAC } \\
\text { ATCGCCTTTTTCGTCCAAAAGGAGTCCCCAGGTTCGATCCGCATGGCGTTGTGCGTGC } \\
\text { GTGCCTTTCTTTTCAAATGATTACGGCTATTAACTTGGGGGCGTTAAGTTGGAAACAC } \\
\text { GTAAATTGCAGACTGCGATTAGAGTGACCATGAGTAGGAGTTCAAAATCTCCTGACA } \\
\text { TCATTTTCTTAAAACCTGCTTTGTTTTTACATTTCTATTTAATATAACTCCTATTTGAAT } \\
\text { AAAAAAACAAAACAAGTTAAGATGTTAAGATATTAACTACATCCTTTGCTCCAAAGGG } \\
\text { AGAGGGGAAGTTATGGAGTTAATTAATTTGCTGTTGGAAATCAATATGGAGTCAGAA } \\
\text { ATATAATGATTTACTAAACCTTATTGAATCGGTAACGATGCGAATTTATATTAAAATA } \\
\text { GCTTTTATGAAACATTCAACAAAAATATTATTAATGTTGGCCCACTTTAGCAACCGGTT } \\
\text { AGGTCTACCGGTTGGGCAAGCAAAGATTCACGCCCTGGTTCGAGTCCCAACTAGTCC } \\
\text { TGCAAAATACCGCAGCAAGTTTAGAGAGACCAAGTGCCATTACCTCTCCCACTTCAG } \\
\text { TTATCGGTTATGCGGCGTTAAGTCGACAGCTTGCCGTCTCTAGCTCCGGTGCCTATA } \\
\text { TAAAGCAGCCCGCTTTCCACATTTCATATTCGTTTTACGTTTGTCAAGCCTCATAGCCG } \\
\text { GCAGTTCGAACGTATACGCTCTCTGAGTCAGACCTCGAAATCGTAGCTCTACACAATT } \\
\text { CTGTGAATTTTCCTTGTCGCGTGTGAAACACTTCCAAGGCGGCCGCCCGACTCTAGAT } \\
\text { CATAATCAGCCATACCACATTTGTAGAGGTTTTACTTGCTTIAAAAAACCTCCCACACC } \\
\text { TCCCCCTGAACCTGAAACATAAAATGAATGCAATTGTTGTTGTTAACTTGTTTATTGCA } \\
\text { GCTTATAATGGTTACAAATAAAGCAATAGCATCACAAATTTCACAAATAAAGCATTTT } \\
\text { TTTCACTGCATTCTAGTTGTGGTTTGCCCAAACTCATCAATGTATCTTAGCGGCTCGAG } \\
\text { GGTACCTCTAG }\end{array}$ \\
\hline $\begin{array}{l}\text { P. Bru Lac } \\
\text { Polyporus } \\
\text { brumalis laccase } \\
\text { gene, codon } \\
\text { optimised for } D \text {. } \\
\text { melanogaster } \\
\text { with overlaps to } \\
\text { pMC-1-1-1 and } \\
\text { larval cuticle } 9 \\
\text { signal peptide } \\
\text { sequence. } \\
\text { Generates pMC- } \\
1-2-6\end{array}$ & $\begin{array}{l}\text { gtgtgaaacacttccaagGCaatcttacaaaATGAAGTTTGTTATTGTTCTGGCTTGCCTGTTGG } \\
\text { CAGTTGTCTTTGCCGCTATTGGCCCTGTTACCGACTTGACGGTTACGGATGCCAACAT } \\
\text { CAGTCCCGACGGATTACGCGCGCCGGTATAGTGGTCAACAACGTGTTCCCCGCTCC } \\
\text { CCTGATTACGGGACAAAAAGGCGACAGGTTTCAGCTGAATCTGGTCAACCAAATGAG } \\
\text { TAATCATACGATGTTGAAGACAACTTCGATACACTGGCACGGTTTTTTCAAAAGGGA } \\
\text { ACTAATTGGGCGGATGGACCCGCATTCGTGAATCAGTGTCCTATCGCTAGCGGTAAT } \\
\text { TCGTTTCTGTATGACTTTCAGGTGCCCGATCAAGCTGGAACTTTCTGGTACCACTCCCA } \\
\text { CCTGTCCACGCAGTATTGTGACGGTCTCCGCGGACCTTTCGTAGTTTATGACCCAACT } \\
\text { GACCCTCACCTGTCGCTGTATGACGTGGATGACGATAGCACAGTTATAACGCTGGCG } \\
\text { GATTGGTACCATGTGGCCGCACGCCTGGGACCGCGCTTCCCGTTGGGTGCTGACTCC } \\
\text { ACTCTGATCAACGGTTTGGGCAGGTCCACCGCGACTCCGACTGCTGATCTGGCTGTG } \\
\text { ATCAGTGTAACCAAAGGTAAACGTTACCGGTTTAGGCTGGTGAGTATTTCCTGCGAT } \\
\text { CCGAACCATACCTTCAGCATTGACGGTCACAAGCTCACCGTCATCGAAGCTGATGGC } \\
\text { ATTTCCACTCAACCCGTCACTGGCATTGACTCCATTCAAATTTTTGCGCACAGAGGTA } \\
\text { CAGCTTCGTCCTCACCGCCGACCAGGATGTAGACAATTACTGGGTTAGGGCTAACCCT } \\
\text { AATTTTGGTACTACTGGATTTCCGGAGGCATTAACAGCGCGATTCTCCGTTATGATG } \\
\text { GTGCACCTGCGGTTGAACCCACTACCTCCCAAACCGGTACGAATCTGCTCGTGGAGA } \\
\text { CAGATCTGCATCCTCTGAGCACAATGCCCGTTCCGGGACTGCCGACACAAGGTGGTG } \\
\text { CCGATTTTAATCTCAATCTCGCCTTAACTTIAACGGTTCGGACTTCTTTATTAACGGT } \\
\text { GCTTCGTTCGTTCCACCGACCGTCCCTGTCCTCCTCCAGATAATCAGCGGTGCGAACA } \\
\text { GCGCCCAAGATTTGCTCCCAAGTGGTTCGGTATATGCACTCCCCTCGAATAGCAGCAT } \\
\text { CGAACTCACATTCCCGGCCACTGCTGCAGCGCCAGGCGCCCCCCATCCATTCCACTTG } \\
\text { CATGGCCACGCTTTTGCCGTAGTACGCAGTGCTGGAAGTACTGTTTATAACTACGACA }\end{array}$ \\
\hline
\end{tabular}




\begin{tabular}{|c|c|}
\hline & $\begin{array}{l}\text { ACCCGGTATTCAGGGATGTAGTTTCCACTGGTACGCCCGCTGCGGGCGACAACGTCA } \\
\text { CCATCCGATTCCAAACGGACAATCCTGGACCATGGTTTTTGCATTGTCACATCGACTT } \\
\text { CCACCTCGATGCTGGCTTCGCTGTCGTGTTCGCGGAAGACCTCCCGGACGTTGTAAGT } \\
\text { GCAAACCCCGTGCCCCAAGCGTGGAGCGATTTGTGCCCCATCTATAACGCTCTCGATC } \\
\text { CGTCGGATCAGTAAGCccgactctagatcataatc }\end{array}$ \\
\hline $\begin{array}{l}\text { Trametes trogii } \\
\text { laccase gene, } \\
\text { codon } \\
\text { optimised for } D \text {. } \\
\text { melanogaster } \\
\text { with overlaps to } \\
\text { pMC-1-1-1 and } \\
\text { larval cuticle } 9 \\
\text { signal peptide } \\
\text { sequence. } \\
\text { Generates pMC- } \\
1-2-3\end{array}$ & $\begin{array}{l}\text { gtgtgaaacacttccaagGCaatcttacaaaATGAAGTTTGTTATTGTTCTGGCTTGCCTGTTGG } \\
\text { CAGTTGTCTTTGCCGCTATTGGTCCAGTTGCAGATCTCACTATCAGCAACGGAGCGGT } \\
\text { TAGTCCTGATGGATTCTCCCGCCAAGCCATATTGGTGAACGATGTGTTCCCGTCGCCG } \\
\text { TTGATAACGGGAAATAAAGGTGACAGGTTTCAGCTCAACGTAATAGACAACATGACA } \\
\text { AATCACACGATGCTCAAATCCACAAGTATCCATTGGCATGGTTTTTCCAACATGGCA } \\
\text { CAAATTGGGCTGATGGCCCTGCATTCGTCAACCAATGTCCCATTAGTACGGGCCACGC } \\
\text { TTTTCTGTATGATTTCAGGTTCCGAATCAGGCGGGTACCTTCTGGTATCACTCGCATT } \\
\text { TGAGCACTCAGTACTGCGACGGCCTGCGTGGTCCAATCGTAGTCTATGATCCCCAGG } \\
\text { ACCCGCATAAAAGTCTGTATGATGTTGATGATGACAGTACAGTGATAACCCTCGCCG } \\
\text { ACTGGTATCATTTGGCAGCTAAGGTCGGCTCGCCGGTACCAACAGCCGACGCAACAC } \\
\text { TCATAAATGGCCTGGGTAGGTCCATAGACACCCTGAACGCAGATTTGGGGTATTA } \\
\text { CCGTTACAAAAGGCAAGAGGTATCGCTTCCGTTTGGTCAGTTTGAGTTGCGACCCAA } \\
\text { ATCATGTTTTCTCCATTGATGGTCATTCGTTGACCGTAATTGAGGCGGATTCCGTAAA } \\
\text { CTTGAAGCCTCAAACGGTAGATAGTATCCAAATCTTTGCGGCCCAACGGTATAGCTTC } \\
\text { GTCCTGAATGCTGATCAAGATGTGGGCAACTACTGGATTCGAGCTCTGCCGAATAGT } \\
\text { GGCACACGCAACTTTGACGGAGGTGTTAATTCGGCGATCCTCCGCTATGATGGCGCC } \\
\text { GCGCCCGTCGAACCGACAACCAGTCAAACGCCCAGCACGAATCCACTCGTAGAGTCG } \\
\text { GCCCTGACAACATTGGAGGGAACAGCAGCGCCGGGTAGCCCTGCTCCGGGAGGTGT } \\
\text { AGACCTCGCTCTGAACATGGCGTTTGGCTTTCTGGCGGCAAGTTACCATAAATGG } \\
\text { AGCAAGCTTTACTCCCCGACCGTCCCTGTGCTGTTGCAGATACTCTCCGGTGCGCAG } \\
\text { TCGGCCCAAGATCTGCTCCCGTCGGGCTCCGTTATCCCTGCCCGCCAATGCAGACA } \\
\text { TCGAAATCAGCTTGCCAGCAACGGCTGCCGCTCCCGGTTTCCACACCCGTTTCATCT } \\
\text { CCACGGTCATACTTTTGCTGTGGTTCGCAGCGCAGGCTCCAGTACATACAATTATGAG } \\
\text { AATCCCGTCTACCGTGACGTCGTTCGACTGGCTCGCCCGGCGATAATGTCACCATCC } \\
\text { GTTTCCGAACGGATAACCCGGGCCCATGGTTTCTGCATTGTCATATAGACTTTCATTT } \\
\text { GGAGGCGGGTTTTGCAGTGGTTATGGCCGAGGATATCCCGGAGGTTGCCGCCACTA } \\
\text { ACCCCGTACCACAAGCATGGTCGGATCTGTGCCCCACATACGACGCGCTGTCGCCGG } \\
\text { ACGATCAGTAAGCCCgactctagatcataatC }\end{array}$ \\
\hline
\end{tabular}

401

402

403

404

405

406

407

408

409

410 
412

413

414

415

416

417

418

419

420

421

422

423

424

425

426

427

428

429

430

431

432

433

434

435

436

437

438

439

440

441

442

443

444

445

446

447

448

449

\section{References:}

1. UN General Assembly. Transforming our world : the 2030 Agenda for Sustainable Development. (2015).

2. Silpa Kaza, Yao, L., Bhada-Tata, P. \& Woerden, F. Van. What a Waste 2.0: A Global Snapshot of Solid Waste Management to 2050. (Urban Dev. World Bank, Washington, DC, 295, 2018).

3. Weitz, M., Coburn, J. B. \& Salinas, E. Estimating National Landfill Methane Emissions: An Application of the 2006 Intergovernmental Panel on Climate Change Waste Model in Panama. J. Air Waste Manage. Assoc. 58, 636-640 (2008).

4. Du, M. et al. Quantification of methane emissions from municipal solid waste landfills in China during the past decade. Renewable and Sustainable Energy Reviews 78, 272279 (2017).

5. Przydatek, G. \& Kanownik, W. Impact of small municipal solid waste landfill on groundwater quality. Environ. Monit. Assess. 191, 1-14 (2019).

6. Landrigan, P. J. et al. The Lancet Commission on pollution and health. Lancet 391, 462-512 (2018).

7. Fuller, R. Hazardous Waste and Toxic Hotspots. in Textbook of Children's Environmental Health 254-261 (Oxford University Press, 2014). doi:10.1093/med/9780199929573.003.0027

8. Lanphear, B. P. et al. Low-level environmental lead exposure and children's intellectual function: An international pooled analysis. Environ. Health Perspect. 113, 894-899 (2005).

9. Kingsford, R. T. et al. Major Conservation Policy Issues for Biodiversity in Oceania. Conserv. Biol. 23, 834-840 (2009).

10. Malaj, E. et al. Organic chemicals jeopardize the health of freshwater ecosystems on the continental scale. Proc. Natl. Acad. Sci. U. S. A. 111, 9549-54 (2014).

11. (CIE), C. of I. E. NSW waste regulation. (2014).

12. Stahel, W. R. The circular economy. Nature 531, 435-438 (2016).

13. Clark, M. \& Maselko, M. Transgene Biocontainment Strategies for Molecular Farming. Frontiers in Plant Science 11, 210 (2020).

14. Lee, J. W., Chan, C. T. Y., Slomovic, S. \& Collins, J. J. Next-generation biocontainment systems for engineered organisms. Nat. Chem. Biol. 14, 530-537 (2018).

15. Maselko, M. et al. Engineering multiple species-like genetic incompatibilities in insects. Nat. Commun. 11, 1-7 (2020).

16. Diener, S., Studt Solano, N. M., Roa Gutiérrez, F., Zurbrügg, C. \& Tockner, K. Biological treatment of municipal organic waste using black soldier fly larvae. Waste and Biomass Valorization 2, 357-363 (2011).

17. Parodi, A. et al. Black soldier fly larvae show a stronger preference for manure than 
for a mass-rearing diet. J. Appl. Entomol. 144, 560-565 (2020).

18. Meyer, H.-P., Minas, W. \& Schmidhalter, D. Industrial-Scale Fermentation. in Industrial Biotechnology 1-53 (Wiley-VCH Verlag GmbH \& Co. KGaA, 2016). doi:10.1002/9783527807833.ch1

19. Dahman, Y., Syed, K., Begum, S., Roy, P. \& Mohtasebi, B. 14 - Biofuels: Their characteristics and analysis. in Woodhead Publishing Series in Composites Science and Engineering (eds. Verma, D., Fortunati, E., Jain, S. \& Zhang Biopolymer-Based Materials, and Bioenergy, X. B. T.-B.) 277-325 (Woodhead Publishing, 2019). doi:https://doi.org/10.1016/B978-0-08-102426-3.00014-X

20. Joosten, L. et al. Review of insect pathogen risks for the black soldier fly (Hermetia illucens) and guidelines for reliable production. Entomol. Exp. Appl. 168, 432-447 (2020).

21. Dortmans, B., Diener, S., Verstappen, B. \& Zurbrugg, C. Black Soldier Fly Biowaste Processing. (2017).

22. Kim, C.-H. et al. Use of Black Soldier Fly Larvae for Food Waste Treatment and Energy Production in Asian Countries: A Review. Processes 9, (2021).

23. Maeda, S. et al. Production of human $\alpha$-interferon in silkworm using a baculovirus vecto. Nature 315, 592-594 (1985).

24. Zeng, S., Zhao, J. \& Xia, L. Simultaneous production of laccase and degradation of bisphenol A with Trametes versicolor cultivated on agricultural wastes. Bioprocess Biosyst. Eng. 40, 1237-1245 (2017).

25. Chivukula, M. \& Renganathan, V. Phenolic Azo Dye Oxidation by Laccase from Pyricularia oryzae. Appl. Environ. Microbiol. 61, 4374 LP - 4377 (1995).

26. Alharbi, S. K. et al. Degradation of diclofenac, trimethoprim, carbamazepine, and sulfamethoxazole by laccase from Trametes versicolor: Transformation products and toxicity of treated effluent. Biocatal. Biotransformation 37, 399-408 (2019).

27. Collins, P. J., Kotterman, M., Field, J. A. \& Dobson, A. Oxidation of Anthracene and Benzo[a]pyrene by Laccases from Trametes versicolor. Appl. Environ. Microbiol. 62, 4563 LP - 4567 (1996).

28. Cañas, A. I. et al. Transformation of Polycyclic Aromatic Hydrocarbons by Laccase Is Strongly Enhanced by Phenolic Compounds Present in Soil. Environ. Sci. Technol. 41, 2964-2971 (2007).

29. Luo, Q. et al. Laccase-Catalyzed Degradation of Perfluorooctanoic Acid. Environ. Sci. Technol. Lett. 2, 198-203 (2015).

30. Luo, Q., Yan, X., Lu, J. \& Huang, Q. Perfluorooctanesulfonate Degrades in a LaccaseMediator System. Environ. Sci. Technol. 52, 10617-10626 (2018).

31. Sumathi, T., Viswanath, B., Sri Lakshmi, A. \& SaiGopal, D. V. R. Production of Laccase by Cochliobolus sp. Isolated from Plastic Dumped Soils and Their Ability to Degrade Low Molecular Weight PVC. Biochem. Res. Int. 2016, 9519527 (2016). 
32. Jin, X. et al. Conditions Optimizing and Application of Laccase-mediator System (LMS) for the Laccase-catalyzed Pesticide Degradation. Sci. Rep. 6, 35787 (2016).

33. Alberts, J. F., Gelderblom, W. C. A., Botha, A. \& van Zyl, W. H. Degradation of aflatoxin B1 by fungal laccase enzymes. Int. J. Food Microbiol. 135, 47-52 (2009).

34. Rodríguez Couto, S. \& Toca Herrera, J. L. Industrial and biotechnological applications of laccases: A review. Biotechnol. Adv. 24, 500-513 (2006).

35. Widsten, P. \& Kandelbauer, A. Laccase applications in the forest products industry: A review. Enzyme Microb. Technol. 42, 293-307 (2008).

36. Witayakran, S. \& Ragauskas, A. J. Synthetic Applications of Laccase in Green Chemistry. Adv. Synth. Catal. 351, 1187-1209 (2009).

37. Kharayat, Y. Distillery wastewater: bioremediation approaches. J. Integr. Environ. Sci. 9, 69-91 (2012).

38. Baghel, S., Sahariah, B. P. \& Anandkumar, J. Bioremediation of Lignin-Rich Pulp and Paper Industry Effluent BT - Combined Application of Physico-Chemical \& Microbiological Processes for Industrial Effluent Treatment Plant. in (eds. Shah, M. \& Banerjee, A.) 261-278 (Springer Singapore, 2020). doi:10.1007/978-981-15-04976_12

39. Ahmed, P. M., Fernández, P. M., de Figueroa, L. I. C. \& Pajot, H. F. Exploitation alternatives of olive mill wastewater: production of value-added compounds useful for industry and agriculture. Biofuel Res. J. 6, 980-994 (2019).

40. Kudanga, T. \& Le Roes-Hill, M. Laccase applications in biofuels production: current status and future prospects. Appl. Microbiol. Biotechnol. 98, 6525-6542 (2014).

41. Viswanath, B., Rajesh, B., Janardhan, A., Kumar, A. P. \& Narasimha, G. Fungal laccases and their applications in bioremediation. Enzyme Res. 2014, (2014).

42. Rochester, J. R. Bisphenol A and human health: A review of the literature. Reproductive Toxicology 42, 132-155 (2013).

43. Chowdhury, M. F. et al. Current treatment technologies and mechanisms for removal of indigo carmine dyes from wastewater: A review. Journal of Molecular Liquids 318, 114061 (2020).

44. Gorman, M. J., Dittmer, N. T., Marshall, J. L. \& Kanost, M. R. Characterization of the multicopper oxidase gene family in Anopheles gambiae. Insect Biochem. Mol. Biol. 38, 817-24 (2008).

45. Waters, A. J., Capriotti, P., Gaboriau, D. C. A., Papathanos, P. A. \& Windbichler, N. Rationally-engineered reproductive barriers using CRISPR \&amp; CRISPRa: an evaluation of the synthetic species concept in Drosophila melanogaster. Sci. Rep. 8 , 13125 (2018).

46. Charles, J. P., Chihara, C., Nejad, S. \& Riddiford, L. M. Identification of proteins and developmental expression of RNAs encoded by the 65A cuticle protein gene cluster in Drosophila melanogaster. Insect Biochem. Mol. Biol. 28, 131-8 (1998). 
47. Almeida, S., Raposo, A., Almeida-González, M. \& Carrascosa, C. Bisphenol A: Food Exposure and Impact on Human Health. Compr. Rev. Food Sci. Food Saf. 17, 15031517 (2018).

48. Atacag Erkurt, H. Biodegradation and Detoxification of BPA: Involving Laccase and a Mediator. CLEAN - Soil, Air, Water 43, 932-939 (2015).

49. Garzillo, A. M. et al. Structural and Kinetic Characterization of Native Laccases from Pleurotus ostreatus, Rigidoporus lignosus, and Trametes trogii. J. Protein Chem. 20, 191-201 (2001).

50. Garzillo, A. M. V et al. Laccase from the white-rot fungus Trametes trogii. Appl. Microbiol. Biotechnol. 49, 545-551 (1998).

51. Atacag Erkurt, H. Biodegradation and Detoxification of BPA: Involving Laccase and a Mediator. CLEAN - Soil, Air, Water 43, 932-939 (2015).

52. Colao, M. C., Lupino, S., Garzillo, A. M., Buonocore, V. \& Ruzzi, M. Heterologous expression of Icc1 gene from Trametes trogii in Pichia pastoris and characterization of the recombinant enzyme. Microb. Cell Fact. 5, 31 (2006).

53. Nakade, K., Nakagawa, Y., Yano, A., Sato, T. \& Sakamoto, Y. Characterization of an extracellular laccase, PbLac1, purified from Polyporus brumalis. Fungal Biol. 114, 609618 (2010).

54. Shmarakov, I. O., Borschovetska, V. L. \& Blaner, W. S. Hepatic Detoxification of Bisphenol A is Retinoid-Dependent. Toxicol. Sci. 157, 141-155 (2017).

55. Bourbonnais, R. \& Paice, M. G. Oxidation of non-phenolic substrates: An expanded role for laccase in lignin biodegradation. FEBS Lett. 267, 99-102 (1990).

56. Morozova, O. V., Shumakovich, G. P., Shleev, S. V. \& Yaropolov, Y. I. Laccase-mediator systems and their applications: A review. Appl. Biochem. Microbiol. 43, 523-535 (2007).

57. Jensen, K. A., Evans, K. M., Kirk, T. K. \& Hammel, K. E. Biosynthetic Pathway for Veratryl Alcohol in the Ligninolytic Fungus Phanerochaete chrysosporium. Appl. Environ. Microbiol. 60, 709-714 (1994).

58. Eggert, C., Temp, U., Dean, J. F. D. \& Eriksson, K.-E. L. A fungal metabolite mediates degradation of non-phenolic lignin structures and synthetic lignin by laccase. FEBS Lett. 391, 144-148 (1996).

59. Felby, C., Nielsen, B. R., Olesen, P. O. \& Skibsted, L. H. Identification and quantification of radical reaction intermediates by electron spin resonance spectrometry of laccase-catalyzed oxidation of wood fibers from beech (Fagus sylvatica). Appl. Microbiol. Biotechnol. 48, 459-464 (1997).

60. Camarero, S., Ibarra, D., Martínez, M. J. \& Martínez, A. T. Lignin-derived compounds as efficient laccase mediators for decolorization of different types of recalcitrant dyes. Appl. Environ. Microbiol. 71, 1775-1784 (2005).

61. Aslam, M. S., Aishy, A., Samra, Z. Q., Gull, I. \& Athar, M. A. Identification, Purification 
and Characterization of a Novel Extracellular Laccase from Cladosporium Cladosporioides. Biotechnol. Biotechnol. Equip. 26, 3345-3350 (2012).

62. Lindsley, D. L., Grell, E. H. \& Bridges, C. B. Genetic Variations of Drosophila melanogaster. Science (80-. ). 162, 993-993 (1968).

63. Venken, K. J. T., He, Y., Hoskins, R. A. \& Bellen, H. J. P[acman]: A BAC transgenic platform for targeted insertion of large DNA fragments in D. melanogaster. Science (80-. ). 314, 1747-1751 (2006). 
